\title{
Estructura interna de la coordinación motriz de los movimientos de pies en ataque del baloncesto \\ Internal structure of the motor coordination of foot movements in attack of basketball
}

\author{
*Noel Fernando Mejia Mejia, **Bergelino Zaldívar Pérez \\ *Universidad Nacional Autónoma de Honduras (Honduras), **Universidad de Ciencias de la Cultura Física y el Deporte \\ "Manuel Fajardo" (Cuba)
}

Resumen. Dentro de los deportes del conjunto, el baloncesto se compone de habilidades abiertas dirigidas a modificar la ejecución de la técnica según las condiciones del entorno. La coordinación motriz es determinante para el control de los gestos técnicos; específicamente para el baloncesto, los movimientos de pies en ataque exigen de un alto control de parte del sistema nervioso central. Cualquier estrategia de enseñanza de la coordinación motriz especifica en los movimientos de pies en ataque requiere del conocimiento de la estructura interna. El objetivo del presente estudio es caracterizar la estructura interna de la coordinación motriz implicada en los movimientos de pies en ataque del baloncesto. Bajo el enfoque cualitativo y el paradigma fenomenológico; los métodos teóricos de análisis-síntesis, inducción-deducción fueron aplicados en conjunto al análisis de contenido y la triangulación de teorías referidas a la coordinación motriz, las capacidades coordinativas y estudios antecedentes. Como resultados mostraron que el baloncesto; específicamente en los movimientos de pies en ataque, son esenciales todas las capacidades coordinativas. Sin embargo, la coordinación visomotriz desempeña un rol determinante en la iniciación y el desempeño deportivo y la coordinación disociada es la que más se manifiesta en las acciones coordinativas; especialmente las que involucran movimientos alternos y simultáneos entre extremidades superiores e inferiores, así como la coordinación entre pie, mano y ojo, como se observa en los movimientos de pies en ataque.

Palabras clave: coordinación disociada, coordinación visomotriz, estructura interna, baloncesto.

\begin{abstract}
Abstrat. In team sports, basketball is composed of open skills aimed at modifying the execution of the technique according to the environmental conditions. Motor coordination is decisive for the control of technical gestures; specifically, for basketball, attacking foot movements require high control from the central nervous system. Any specific motor coordination teaching strategy in attacking foot movements requires knowledge of the internal structure. The objective of the present study is to characterize the internal structure of the motor coordination involved in the movements of the feet in basketball attack. Under the qualitative approach and the phenomenological paradigm; The theoretical methods of analysis-synthesis, inductiondeduction were applied together to the content analysis and the triangulation of theories related to motor coordination, coordinative capacities and antecedent studies. As results they showed that basketball; specifically, in the movements of the feet in attack, all the coordinative capacities are essential. However, visual-motor coordination plays a decisive role in sports initiation and performance, and dissociated coordination is the one that is most manifested in coordinative actions; especially those that involve alternate and simultaneous movements between upper and lower extremities, as well as coordination between foot, hand and eye, as observed in the movements of the feet in attack.
\end{abstract}

Key words: dissociated coordination, visual-motor coordination, internal structure, basketball.

\section{Introducción}

Pias, Camacho y Barrero (2020) explican que, en los deportes de conjunto, especialmente en las modalidades que se desarrollan en entornos inestables, se requieren habilidades abiertas; donde la anticipación y adaptación condicionan el resultado de las acciones técnicas y tácticas. Considerando lo anterior, el baloncesto se reviste de un alto grado de complejidad en el que las

Fecha recepción: 20-03-21. Fecha de aceptación: 31-05-21

Noel Mejia

nmejia@unah.edu.hn variables que inciden en el juego son tan numerosas como las relaciones entre las mismas; aspecto que determina la variabilidad de la técnica deportiva. Tal variabilidad implica un cambio permanente en la ejecución del gesto deportivo, por lo que se requiere de una alta e eficiente percepción que permitan al atleta adaptarse al entorno y producir una respuesta motriz (gesto deportivo) acorde (Dandan, Cruz \& Ballesteros, 2019).

Con el fin de desarrollar las metodologías de enseñanza-aprendizaje en un determinado deporte, aparte de las mencionadas características externas, deben considerarse el conjunto de elementos que conforman los fundamentos técnicos, a través de los cuales se realizan 
las acciones individuales y colectivas. Esto es a lo que Costoya (2002) denomina estructura interna, al respecto el autor indica que:

Los elementos que componen su estructura interna y las relaciones existentes entre ellos cobran vital importancia, de tal modo que describir los unos y las otras es requisito indispensable para poder establecer un criterio de interpretación primero, de estructuración después, y de intervención finalmente. (p. 79)

Uno de los fundamentos técnicos del baloncesto cuya estructura interna es de interés, sin embargo muy poco estudiado, son los movimientos de pies en ataque. Estos fundamentos técnicos permiten iniciar o detener el desplazamiento del jugador en ataque con o sin la posesión del balón (Gonzales, 2019), al tiempo que posibilita hacer frente a las limitaciones impuestas por el reglamento de juego, especialmente a los desplazamientos del jugador en posesión del balón. De esta manera la correcta ejecución de los movimientos de pies en ataque reduce las violaciones al reglamento; aspecto esencial cuando se tiene en cuenta que un objetivo primordial en el baloncesto es mantener la posesión del balón y reducir las pérdidas del mismo.

Por otra parte, el baloncesto es un deporte altamente demandante en el nivel de coordinación motriz requerido para la ejecución de los fundamentos técnicos (Zwierko, Lesiakowski \& Florkiewicz, 2005), esto incluye a los movimientos de pies en ataque, ya que hay una fuerte conexión de los movimientos coordinados entre las extremidades superiores e inferiores (Cortis, Tessitore, Lupo, Pesce, Fossile, Figura, \& Capranica, 2011), implicando al sistema nervioso, responsable del procesamiento de la información propioceptiva, de la musculatura; estructura efectora del movimiento (Mejía \& Zaldívar, 2020), así como del «control neuronal necesario para el mantenimiento de una relación estable de las fases entre el movimiento de segmentos con diferentes características mecánicas.» (Cortis, et al., 2011, p. 135).

Es bien sabido que con la adquisición de la coordinación motriz se facilita el aprendizaje de gestos deportivos de mayor complejidad (Herrera, Valencia, García, \& Echeverri, 2020), y se llega al alto refinamiento y elevado control de los movimientos, permitiendo una ejecucion eficaz y eficiente (Weineck, 2016). Sin embargo, con el fin fundamentar la práctica pedagógica dirigida al aprendizaje de la coordinación motriz implicada en los movimientos de pies en ataque, es necesario conocer la estructura interna del mencionado gesto técnico, aspecto hasta ahora no estudiado.
El objetivo del presente estudio es caracterizar la estructura interna de la coordinación motriz implícita en los movimientos de pies en ataque del baloncesto. Contribuyendo a crear las bases que permitan a los entrenadores y docentes desarrollar las estrategias de enseñanza-aprendizaje de las habilidades relacionadas a este fundamento técnico (Nabaskues, Usabiaga, MartosGarcía, \& Førland, 2019).

\section{Definición y tipología de la coordinación mo-} triz

A pesar que el estudio de la función de la coordinación motriz suele descuidarse al punto de marginarla al olvido (Ruiz, Barriopedro, Ramón, Palomo, Rioja, García \& Navia, 2017), la misma es la base del dominio deportivo y uno de los aspectos más relevantes en la competencia motriz. Esta se refiere al ordenamiento de una serie de acciones, identificándose variables como: ordenamiento muscular y la alternancia agonista antagonistas; la coordinación neuromuscular; el ordenamiento de las fuerzas (sinergias) internas (Meinel, 1987).

De esta manera en la coordinación motriz, con la amplia participación del sistema nervioso, se organizan los procesos parciales de un acto motor; administrando las fuerzas internas y externas por parte de los sistemas reguladores y efectores del movimiento. Siendo la relajación muscular uno de las variables más determinantes en el acto motor (Verkhoshansky, 2018), considerando también la importancia del objetivo deportivo en las acciones técnicas-deportivas (Cenizo, Ravelo, Morilla, Ramírez \& Fernández, 2016).

En la coordinación motriz se expresan conjuntamente las capacidades coordinativas, que son el fundamento de la coordinación motriz (Weineck, 2016), y condicionantes para la ejecución de gestos motores concretos (Martin, Carl \& Lehnertz, 2016). En ellas se experimenta una participación conjunta y no aislada;

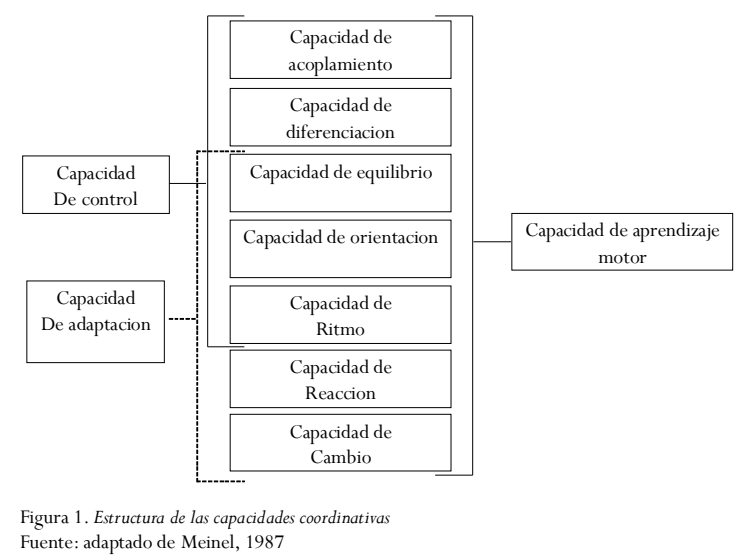


donde, de acuerdo a las particularidades de la modalidad deportiva, unas capacidades son más determinantes que otras. Lo anterior se resume en la figura 1, en la que se evidencia la presencia de dos complejos de capacidades coordinativas: las capacidades que regulación (conducción / control); propias de las modalidades deportivas compuestas de acciones estandarizadas. Estas permiten el control de patrones como amplitud y velocidad del movimiento. Mientras que las capacidades de cambio y adaptación, presentes en los deportes de conjunto con un entorno cambiante de juego, permiten modificar el gesto motor acorde a la situación.

Respecto a las capacidades coordinativas, Mejia (2020) destaca las variables e indicadores más significativos de las mismas:

Capacidad de acoplamiento: se unifican las habilidades motrices independientes, se combinan movimientos aislados de distintos segmentos corporales en la ejecución global del movimiento (Martin et al. 2016), la fluidez es un indicador de una buena capacidad de acoplamiento (Hafelinger \& Schuba, 2010), la cual se concreta con la automatización del gesto motor (Dorochenko, Navarro, Moya, Pérez, Muñoz \& Pérez, 2017).

Capacidad de diferenciación kinestésica: armoniza y diferencia los parámetros de fuerza, tiempo y espacio en cada una de las fases del movimiento (Martin, Nicolaus, Ostrowski \& Rost, 2004), minimizando las variaciones en cada repetición del gesto motor. Se estima la importancia de los propioceptores en el análisis de los patrones de fuerzas, tiempo y espacio (Hafelinger \& Schuba, 2010).

Capacidad de equilibrio: mantiene el equilibrio corporal ante cambios leves o bien repentinos, además potencia la sincronización de los movimientos (Cañizares \& Carbonero, 2016).

Capacidad de orientación espacio-temporal: «determina y modifica la situación y los movimientos del cuerpo en el espacio y en el tiempo, en relación con un campo de acción definido» (Meinel, 1987, p. 252), requiriendo una alta participación de los analizadores ópticos, acústicos y cinestesicos.

Capacidad de ritmo: procesa un ritmo exterior y lo reproduce en movimiento a la vez que internaliza un ritmo propio en la ejecución de los movimientos (Meinel, 1987), organizando «cronológicamente las prestaciones musculares en el espacio y el tiempo» (Cañizares \& Carbonero, 2016, p.12).

Capacidad de reacción: permite movimientos breves y rápidos en respuesta a diversas señales (Martin et al., 2016), aspecto esencial en las modalidades de entornos constantemente cambiantes (Meinel, 1987).

Capacidad de cambio o adaptación: modifica el programa motor a las condiciones del entorno, para ello la percepción y la anticipación son dos variables determinantes en la planificación por anticipado de las acciones necesarias en respuesta a los cambios del entorno (Hafelinger \& Schuba, 2010).

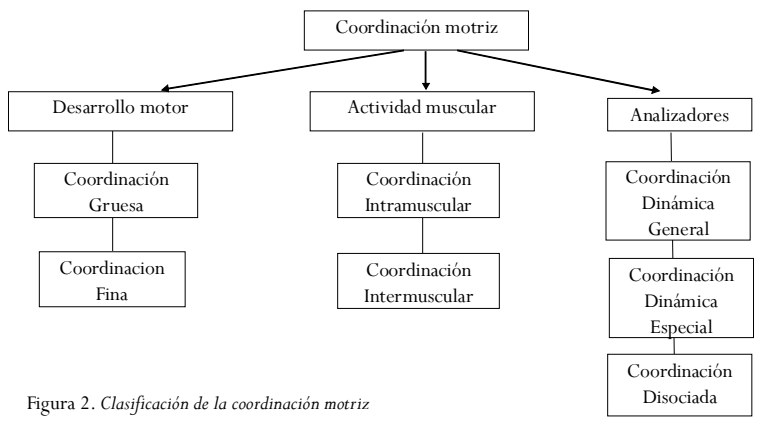

Por otra parte, la coordinación motriz es objeto de diversas denominaciones y clasificaciones, en figura 2 se aprecia la clasificación que Mejía (2020) hace de la coordinación motriz, basado en criterios como el desarrollo motor, la actividad muscular, los analizadores y segmentos corporales involucrados en el movimiento. A continuación, se exponen dichas denominaciones.

Coordinación gruesa y coordinación fina: son la interacción de grandes grupos musculares para producir el desplazamiento en el espacio (coordinación gruesa) y el dominio de los movimientos coordinados de brazos, pero sobre todo de manos y dedos en la manipulación de objetos (coordinación fina) (Serrano \& De Luque, 2019).

Coordinación intramuscular e intermuscular: determina el grado de contracción de un solo músculo, dependiente del número de fibras reclutadas sincrónicamente por las unidades motoras (intramuscular) y la interacción entre la musculatura agonista y la musculatura antagonista (intermuscular) que, mediante la inhibición reciproca permite el movimiento en cuestión (Guyton \& Hall, 2016).

Coordinación dinámica general y coordinación segmentaria: la coordinación dinámica general se refleja en el funcionamiento global de los segmentos corporales (Bernal, J. Wanceulen, J \& Wanceulen, A, 2019a), mientras que la coordinación segmentaria se manifiesta en las acciones motrices que se dan como respuesta a un estímulo visual, y cuya finalidad es la adaptación del movimiento ante el estímulo (Bernal, J. Wanceulen, J \& Wanceulen, A, 2019b). En ese sentido la coordinación segmentaria relaciona distintos segmentos corporales 
(brazos, piernas) dirigidos por el sentido de la vista para el logro de un objetivo, aumentando la complejidad de la acción cuando se involucra la manipulación de un objeto, como el caso de un balón (Cogolludo \& Humanes, 2019).

Coordinación óculo-mano y óculo-pie: es parte de la coordinación segmentaria y se define como la capacidad de «manejar eficazmente y al mismo tiempo un segmento corporal y un móvil, precisando de la interacción y el control de la vista (Wanceulen, A Wanceulen, J. \& Wanceulen, A., 2019, p. 399).

Coordinación disociada: permite realizar un movimiento con un segmento corporal, mientras el otro permanece estático o se mueve en dirección contraria, reflejándose en movimientos coordinados entre manomano, pie-pie y mano-pie. Esta es «la culminación de una coordinación ajustada y completa» (Villada \& Vizuete, 2003, p. 235).

\section{Estudio de la coordinación motriz en el balon-} cesto

En su estudio del 2011, Cortis et al., señalaron las conexiones existentes entre manos, pies, ojos y el balón en las acciones técnicas; resaltando el control neuronal para mantener la relación entre distintos segmentos corporales; aspectos inherentes a la coordinación disociada.

Posteriormente en el 2014; Jerzy, PaweB, Janusz, Tomasz, \& Mariusz, describieron la estructura de la coordinación motriz en el baloncesto, enfatizando en la capacidad de diferenciación kinestésica, la capacidad de orientación espacio-temporal, la capacidad de reacción, la capacidad de acoplamiento, la capacidad de ritmo, y la capacidad de adaptación. Igualmente, Andrieieva, Yarmak, Kyrychenko, Ravliuk, Tsurka, Zavgorodnia, Strazhnikova \& Potop, (2020) identificaron a la orientación espacio-temporal, el equilibrio dinámico y estático como las cualidades coordinativas más determinantes en niños de 12 años de edad que practicaban el baloncesto.

De esta manera se resalta la significancia del entrenamiento de las capacidades coordinativas en el proceso de preparación deportiva, para lo cual y según los resultados del modelo factorial desarrollado por Boichuk, Iermakov, Kovtsun, Pasichnyk, Melnyk, Lazarenko \& Troyanovska, (2018), se debe entrenar los factores más determinantes de la coordinación motriz en el baloncesto, como ser: la reconstrucción rápida de la acción motriz, la diferenciación kinestésica, la reacción compleja y la orientación espacial.
Por su parte, Kasabova (2019), mediante el análisis factorial comparo jugadores experimentados versus no experimentados. Identifico a la coordinación especifica como el factor más importante en los jugadores experimentados, mientras que, en los jugadores menos experimentados, el principal factor fue la velocidad y la fuerza, siendo las cualidades coordinativas el segundo factor. Tal diferencia se explica desde la consideración de la edad como factor condicionante en la maestría de los gestos deportivo de mayor complejidad (Boichuk, et al., 2018; Andrieieva, et al., 2020). Lo anterior, coincide con lo expuesto por Irawan \& Lesmana (2019), quienes manifiestan que en las fases iniciales del aprendizaje el sentido de la vista es el principal analizador implicado en el control de los movimientos, no así en las fases avanzadas de maestría deportiva, donde los propioceptores, responsables de las sensaciones quinestésicas, median en los gestos deportivos de alta intensidad. No es hasta que se alcanza un elevado nivel de sensibilidad en los receptores de la piel (mecanorreceptores); un alto desarrollo de los sensores vestibulares; y un alto dominio de la coordinación inter e intra muscular, que pueden controlarse los movimientos deportivos con fluidez y claridad (Mejia \& Zaldívar, 2020).

\section{Metodología}

El presente estudio se dirigió bajo el enfoque cualitativo, en conjunto con el análisis de contenido; técnica que permite la interpretación de la información: escrita, visual, etc. Al respecto Hostil (1969) manifiestan que «El análisis de contenido es una técnica de investigación para formular inferencias identificando de manera sistemática y objetiva ciertas características específicas dentro de un texto» (p.5).

\section{Objeto de análisis}

Como objeto de análisis se establece la coordinación motriz. Para ello se desarrolló una investigación conceptual, estableciendo como unidades de análisis y registro las definiciones referidas a: la coordinación motriz, las siete (7) capacidades coordinativas, la coordinación segmentaria (visomotriz) y la coordinación disociada.

\section{Codificación}

Se identificaron los conceptos y términos utilizados en las definiciones procedentes de distintos autores. Se utilizó como reglas de recuento la presencia de dichos 
conceptos y términos referidos a: la coordinación motriz, las siete (7) capacidades coordinativas, la coordinación segmentaria (visomotriz) y la coordinación disociada.

\section{Categorización}

Los términos y conceptos identificados fueron agrupados en las categorías ya predefinidas y correspondientes a los movimientos de pies: paradas, salidas, pivotes, fintas y cambio de dirección.

\section{Resultados}

La revisión teórica y análisis conceptual permitió identificar los indicadores y unidades de análisis (Tabla 1) que fueron integradas a la construcción de la estructura interna que refleja los elementos de la coordina-

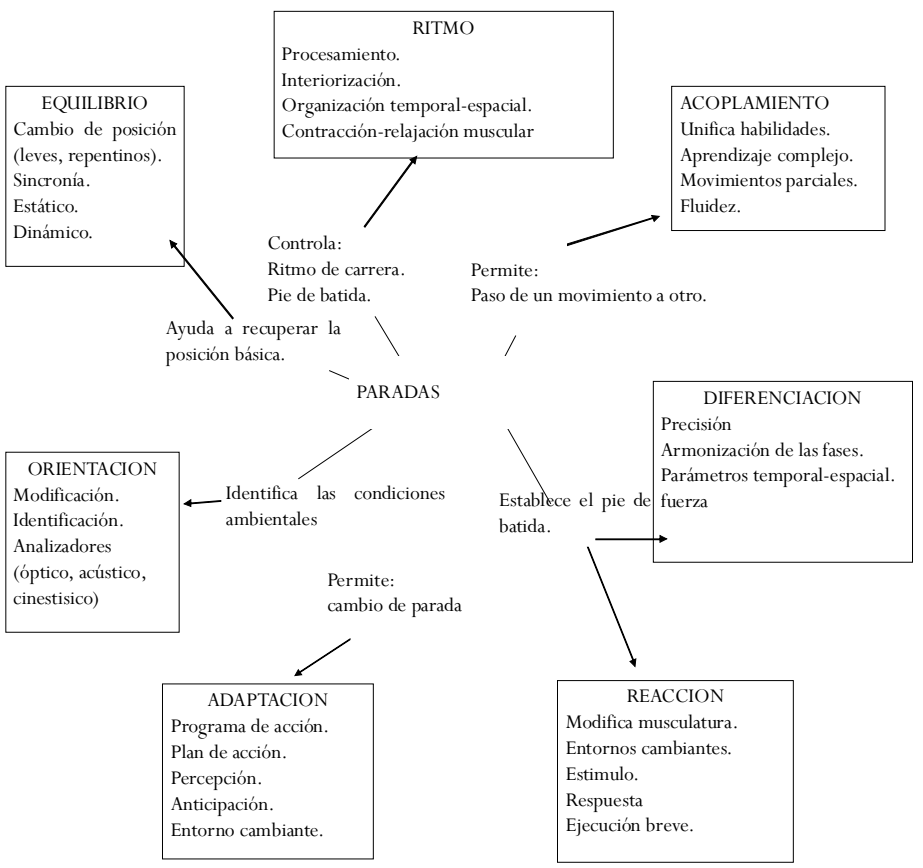

Figura 3. Las capacidades coordinativas en la estructura interna de las paradas. ción motriz presentes en los movimientos de pies en ataque del baloncesto. Seguidamente se especifican dichos elementos.

capacidad de ritmo y la capacidad de cambio permiten establecer un ritmo en la fase de carrera y modificar el mismo. Ambas capacidades, junto con la capacidad de

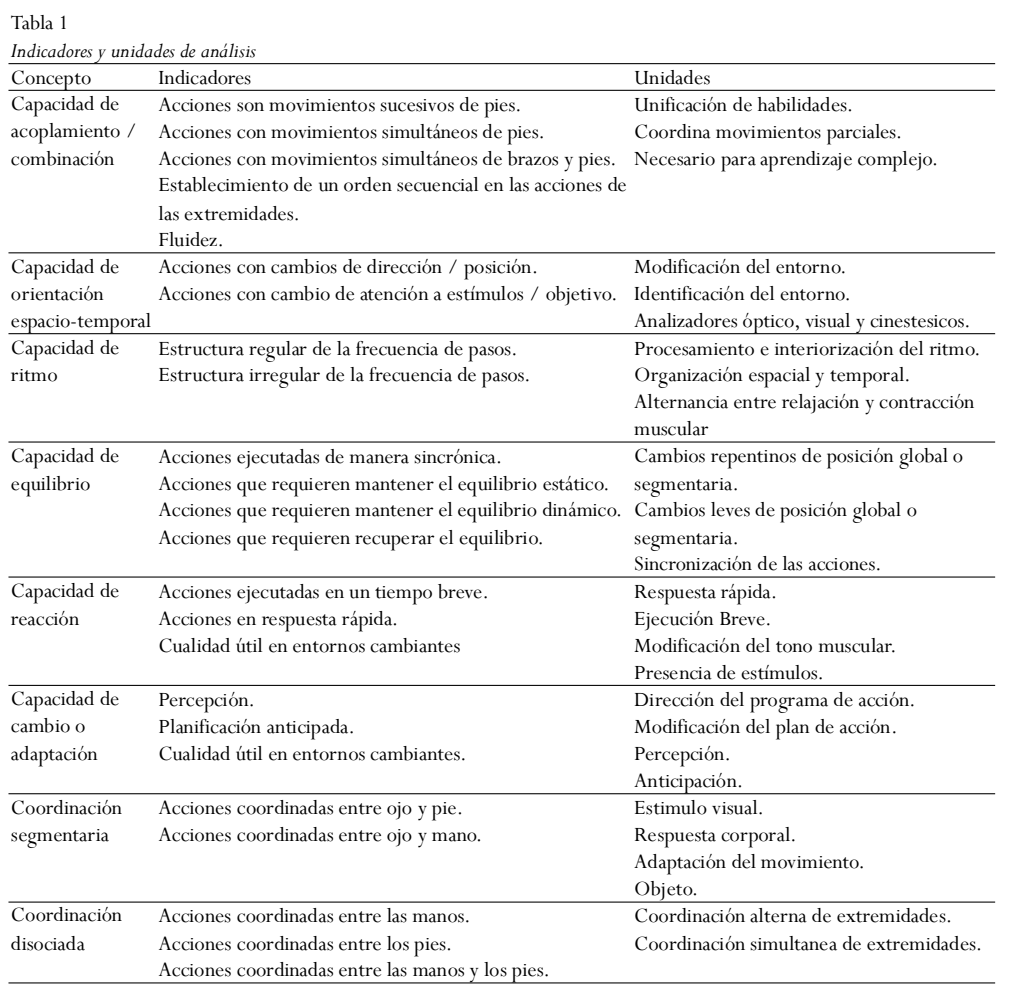
reacción y de diferenciación son esenciales para establecer el pie con que el jugador realizara la batida, lo que a su vez determinara el pie pivote y las posibilidades de desplazamiento a partir de este último. De la misma forma la orientación espacio-temporal determina con que pie es más adecuado realizar la batida, de acuerdo al espacio de la cancha donde se encuentra y la posición de los jugadores contrarios. La capacidad de equilibrio estático y dinámico ayudan a mantener y recuperar la postura básica en la fase de caída, sobre todo para detención de los desplazamientos a alta velocidad, así como en la parada a un tiempo. En cuanto a la coordinación motriz, en la figura 4 se observa que la coordinación óculo-mano y óculo-pie (coordinación segmentaria) están presentes en la

Las paradas: estas permiten la detención del desplazamiento con y sin balón y se dividen en detenciones de uno y dos tiempos. En su ejecución se realiza un pequeño salto para luego caer en la postura básica, comúnmente las detenciones se realizan en cuatro fases (carrera, batida, vuelo y caída).

La figura 3 muestra la estructura interna de las paradas: en lo que respecta a las capacidades coordinativas, la recepción del balón; y la coordinación de mano-pie homolateral y contralateral (coordinación disociada) regula las detenciones después del bote.

Las salidas: estas son abiertas y cruzadas, representan los movimientos realizados por el jugador estático en posesión del balón para iniciar su desplazamiento teniendo como objetivo adquirir cierta ventaja sobre el adversario. Para su ejecución se requiere liberar el pie 
de salida del peso corporal, trasladándolo hacia la pierna de impulso, posteriormente el peso del cuerpo se envía al pie con el que se avanza en la salida.

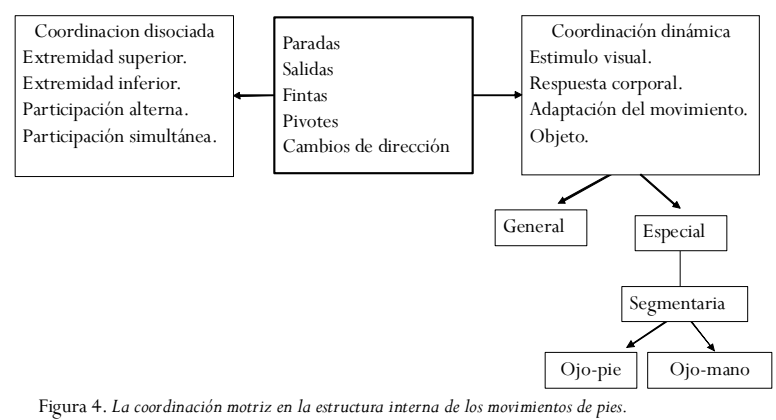

La figura 5 muestra la estructura interna de las salidas: dentro de las capacidades coordinativas, la capacidad de acoplamiento contribuye a coordinar los movimientos parciales de brazos y piernas y la capacidad de orientación espacio-temporal determina la dirección a seguir y el momento de la realización de la salida. En la coordinación motriz, la figura 4 muestra como en la salida abierta se manifiesta la coordinación mano-pie homolateral y en la salida cruzada la coordinación manopie contralateral (coordinación disociada).

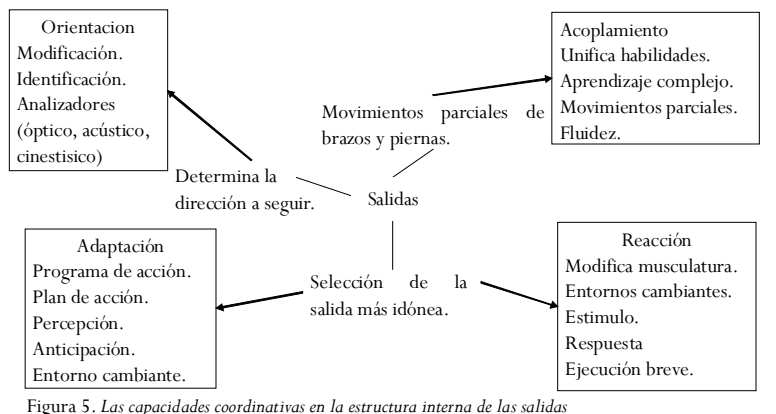

Los pivotes: estos son el pivote anterior y el pivote posterior, permiten al jugador movilizarse alrededor de un eje longitudinal, orientando su cuerpo hacia un objetivo o dirección determinada, este gesto motor se realiza con un pie fijo en el suelo (pie pivote) y el otro en movimiento. El reglamento impide que se cambie, deslice o se levante el pie pivote hasta haber soltado el balón, bien sea mediante el bote, el pase o el tiro.

La figura 6 muestra la estructura interna de las salidas: dentro de las capacidades coordinativas son fundamentales la capacidad de orientación y la lateralidad para poder identificar hacia qué lado girar y que pivote es el más conveniente. El movimiento vertiginoso del giro sobre el eje longitudinal rompe la condición de equilibrio por lo que la capacidad de equilibrio es necesaria para mantener dicho estado o recuperarlo. La capacidad de cambio y la capacidad de combinación son requeri- das para dar continuidad a otros gestos motores después del pivote; como por ejemplo la salida.

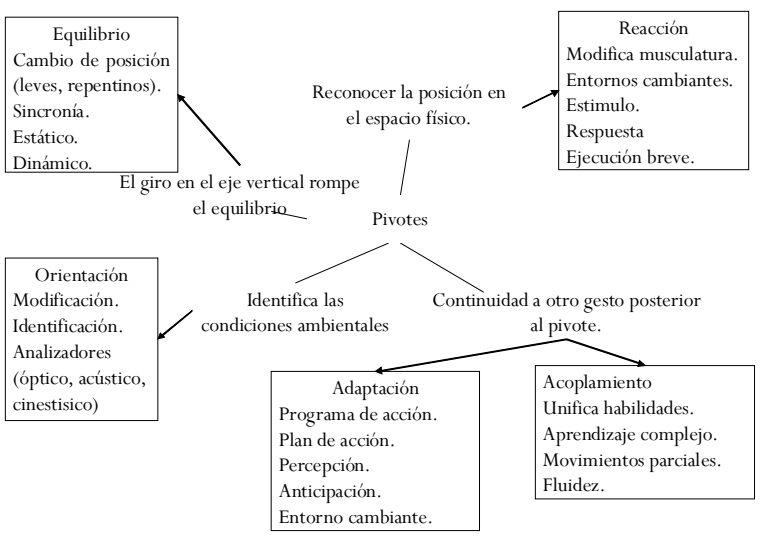

Figura 6. Las capacidades coordinativas en la estructura interna de los pivotes

Las fintas: estas son las fintas de tiro, de pase y de salida; en especial las fintas de salida, consisten en engañar al adversario iniciando un gesto o movimiento hacia una dirección para posteriormente tomar la dirección opuesta a la inicial. La finta de salida está condicionada por la estructura interna de las salidas y los pivotes (Figura 5 y 6 ).

Los cambios de dirección: estos son normal y reverso, consisten en cambiar la dirección del desplazamiento de forma explosiva y sorpresiva, pudiendo incluir un cambio de ritmo (aceleración). En su ejecución se detiene el desplazamiento con una ligera parada, se pivota en la nueva dirección y realiza una salida, imprimiendo un cambio de ritmo. En este fundamento se amalgaman todos los movimientos de pies en ataque mencionados anteriormente y cada una de las capacidades coordinativas implicadas en los mismos.

\section{Discusión}

En su estudio del 2014, William, Shirley, Yoyo, Dinisha, Hiu Yan Lau, \& Carina reconocen la importancia de una excelente coordinación ojo-mano (segmentaria), sobre todo en las acciones que requieren movimientos de pivote, combinación de movimientos de pies, equilibrio, agilidad y cambios de dirección en los deportes con entornos altamente desafiantes, como el baloncesto. Tales acciones pueden ser observadas en los movimientos de pies en ataque, como ser el caso de la salida cruzada; cuando el jugador pivota sobre uno de los pies y combina el movimiento de ambos pies junto con la acción de la mano que dirigirá el bote del balón, al tiempo que se realiza un cambio de dirección.

El rol de la coordinación ojo-mano es desglosado por Candra (2019) quien expone que en la ejecución de un 
movimiento el ojo es el órgano visual que provee información, mientras que las manos ejecutan la acción. En el ejemplo citado anteriormente, el ojo percibe la posición del pie de salida para evitar que la acción de bote de la mano (opuesta) envíe el balón al pie.

Las estructuras internas expuestas coinciden con los resultados reportados por Zwierko, et al., (2005), donde se señalan una serie de habilidades coordinativas necesarias para el desempeño eficiente en el baloncesto, entre estas se encuentran la orientación espacial, la reacción, el ritmo, el equilibrio y el enlace de movimientos parciales. Poniendo como ejemplo una parada en dos tiempos para realizar una recepción del balón; la misma no sería posible si, los movimientos parciales implicados en dicha acción no fuesen coordinados por la capacidad de combinación, como por la coordinación disociada. Igualmente, los autores en mención, indican que «el nivel de frecuencia de movimiento en los jugadores de baloncesto se traduce en una alta capacidad coordinativa.» (p. 127), este es un aspecto sumamente decisivo en el baloncesto, ya que permite acelerar o desacelerar a fin de controlar la velocidad que posibilite ganar ventaja sobre el adversario (Valle \& Vila, 2015), así la frecuencia de pasos requiere de la alternancia entre un ritmo regular e irregular.

De la misma manera que la coordinación motriz potencia el aprendizaje de los movimientos de pies en ataque, estos últimos pueden potenciar la eficiencia y eficacia en la ejecución de otros fundamentos técnicos tales como pasar, botar, lanzar. Así lo sustenta el estudio de Kumar (2014), donde el grupo que realizo entrenamiento coordinativo para las extremidades inferiores mejoro sustancialmente en los fundamentos técnicos antes mencionados, por lo que se deduce que todo fundamento técnico requiere de una solidad base coordinativa de movimientos de pies.

\section{Conclusiones}

En el baloncesto, específicamente en los movimientos de pies en ataque son esenciales todas las capacidades coordinativas; sin embargo, la coordinación visomotriz (ojo-mano, ojo-pie) desempeña un rol determinante en la iniciación y el desempeño deportivo, y la coordinación disociada es la que más se manifiesta en las acciones coordinativas; especialmente las que involucran movimientos alternos y simultáneos entre extremidades superiores e inferiores, así como la coordinación entre pie, mano y ojo, como se observa en los movimientos de pies en ataque.

\section{Referencias}

Andrieieva, O., Yarmak, O., Kyrychenko, Ravliuk, T., Tsurka, T., Zavgorodnia, T., Strazhnikova, I. \& Potop, V. (2020). The factor structure of physical and motor fitness of 12-year-old children while playing basketball. Journal of Physical Education and Sport, 20 (3), 1613-1620.

Bernal, J., Wanceulen, J. \& Wanceulen, A. (2019a). 200 juegos y ejercicios de coordinación óculo manual. España: Editorial Wanceulen.

Bernal, J., Wanceulen, J. \& Wanceulen, A. (2019b). 400 Juegos y Ejercicios de Imagen y Percepción Corporal. España: Editorial Wanceulen.

Boichuk, R., Iermakov, S., Kovtsun, V., Pasichnyk, V., Melnyk, V., Lazarenko, M. \&Troyanovska, M. (2018). Individualization of basketball players (girls) coordination preparation at the stage of preparation for the highest achievements. Journal of Physical Education and Sport. 18(3), $1722-1730$.

Candra, O. (2019). The Contribution of Eye-Hand Coordination to Basketball Lay Up Shoot Skills. Advances in Social Science, Education and Humanities Research, 464, 864-869.

Cañizares, J. \& Carbonero, C. (2016). Enciclopedia de educación física en la edad escolar: Colección manual para el profesorado de educación física en la edad escolar. España: Editorial Wanceulen.

Cenizo, J., Ravelo, J., Morilla, S., Ramírez, J. \& Fernández, J. (2016). Diseño y validación de instrumento para evaluar coordinación motriz en primaria. Revista Internacional de Medicina y Ciencias de la Actividad Física y del Deporte, 16 (62), 203-219.

Cogolludo, E. \& Humanes, A. (2019). Valoración de la condición física e intervención en accidentes. España: EDITX.

Cortis, C., Tessitore, A., Lupo, C., Pesce, C., Fossile, E., Figura, F. \& Capranica, L. (2011). Inter-limb coordination,strength,jump, and sprint performances following a youth men's basketball game. Journal of Strength and Conditioning Research, 25 (1), 135142.

Costoya, R. (2002). Baloncesto: metodología del rendimiento. España. INDE.

Dandan N., Cruz, C. \& Ballesteros, T. (2019). Perceived Level of Emotional Intelligence and Sport Performance of College Varsity Athletes in Open-Skill and Closed-Skill Sports. Research on Humanities and Social Sciences. 9 (12), 10-17.

Dorochenko, P., Navarro, S., Moya, I., Pérez, D., Muñoz, 
J. \& Pérez, M (2017). Coordinación y equilibrio en el pádel. España: Editorial Wanceulen.

Gonzales, J. (2019). Los movimientos básicos en el baloncesto (juego de pies). Rescatado de http:// angeljareno.es/tecnica/los-movimientos-basicos/.

Guyton, A. \& Hall, J. (2016). Tratado de Fisiología Médica $13^{a}$ Edición. España: Elsevier, S.A.

Hafelinger, U. \& Schuba, V. (2010). La coordinación y el entrenamiento propioceptivo. Barcelona: Editorial Paidotribo.

Herrera, B., Valencia, W., García, D. y Echeverri, J. (2020). Desarrollo de las capacidades coordinativas en niños: efectos de entrenamiento en el patinaje. Retos, 38, 282-29.

Hostil, O. (1969). Content analysis for the social sciences and humanities. Addison.

Irawa, R. \& Lesmana, S. (2019). Validity and Reliability Testing on Eye Hand Coordination Basketball Players «Overhead and Under Arms Throw». Advances in Social Science, Education and Humanities Research, 464, 447-449.

Jerzy, S., PaweB, W., Janusz, Z., Tomasz, N. \& Mariusz, B. (2014). Structure of coordination motor abilities in male basketball players at different levels of competition. Pol. J. Sport Tourism, 21, 234-239.

Kasabova, L. (2019). Structure of motor opportunities of basketball students - with different basketball experience. KNOWLEDGE - International Journal 34 (6), 1801-1806.

Kumar, N. (2014). Effect of basketball specific footwork training protocol on selected offensive and defensive skills in basketball. International Journal of Physical Education, Fitness and Sports. 3 (2), 60-67.

Martin, D., Carl, K. \& Lehnertz, K. (2016). Manual de metodología del entrenamiento deportivo. Barcelona: Editorial Paidotribo.

Martin, D., Nicolaus, J., Ostrowski, C. \& Rost, K. (2004). Metodología general del entrenamiento infantil y juvenil. Barcelona: Editorial Paidotribo.

Meinel, K. (1987). Teoría del movimiento. Buenos Aires: Stadium S. R. L.

Mejía Mejía, N.F. (2020). Revisión conceptual y tipología de la coordinación motriz.

Mejía, N. \& Zaldívar, B. (2020). Bases neurológicas para el aprendizaje y entrenamiento de la técnica deportiva. Acción, 16, 1-10.

Nabaskues Lasheras, I., Usabiaga Arruabarrena, O., Martos-García, D. y Førland Standa, Ø. (2019). Comprensión de la habilidad desde la perspectiva del futuro profesorado de Educación Física. Retos,
36, 121-128.

Pias, D., Camacha, P. y Barrero, A. (2020). La anticipación en lo deportes de equipo: los procesos perceptivos para su mejora. España: Editorial Wanceulen.

Ruiz, L., Barriopedro, M., Ramón, I., Palomo, M., Rioja, N., García, V. \& Navia, J. (2017). Evaluar la Coordinación Motriz Global en Educación Secundaria: El Test Motor SportComp. Revista Internacional de Ciencias del Deporte, 13 (49), 285-301.

Serrano, P. \& De Luque, C. (2019). Motricidad fina en niños y niñas: Desarrollo, problemas, estrategias de mejora y evaluación. España: Narcea Ediciones.

Valle, S y Vila, C. (2015). Ejercicios generales y específicos para la mejora de la coordinación de pies aplicada al baloncesto. Rescatado de https:// www.fbcv.es/blog/2015/01/ejercicios-generales-yespecificos-para-la-mejora-de-la-coordinacion-depies-aplicada-al-baloncesto-y-4/.

Verkhoshansky, Y. (2018). Teoría y metodología del entrenamiento deportivo. Barcelona: Editorial Paidotribo.

Villada, P. y Vizuete, M. (2003). Los fundamentos teóricos-didácticos de la educación física. Madrid: Secretaria General Técnica.

Wanceulen, A., Wanceulen, J. \& Wanceulen, A. (2019). Gestión y dirección deportiva del futbol formativo. España: Editorial Wanceulen.

Weineck, J. (2016). Entrenamiento total. España. Editorial Paidotribo.

WilliamW. N.Tsang, Shirley S. M. Fong,Yoyo T.Y. Cheng, Dinisha D. Daswani, Hiu Yan Lau, Carina K. Y. Lun, Shamay S. M. Ng. (2014). The Effect of Vestibular Stimulation on Eye-Hand Coordination and Postural Control in Elite Basketball Players. American Journal of Sports Science. 2 (2), 17-22.

Zwierko, T., Lesiakowski, P. y Florkiewicz, B. (2005). Selected aspects of motor coordination in young basketball players. Human Movement, 6 (2), 124-128.

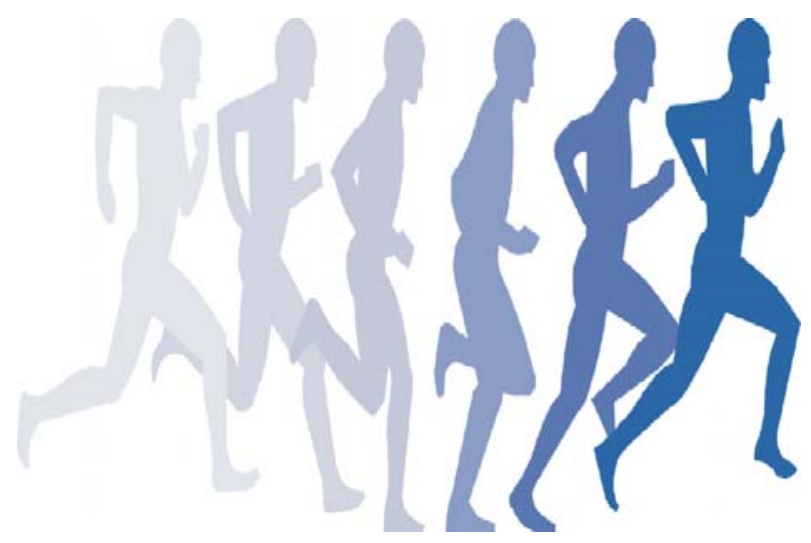

